\title{
Exploring Natural Allelic Variations of the $\beta$ - Triketone Herbicide Resistance Gene HIS1 for Application in indica Rice and Particularly in Two-Line Hybrid Rice
}

Qiming Lv ${ }^{1,2+}$, Xiuli Zhang ${ }^{1,2+}$, Dingyang Yuan ${ }^{1,2+}$, Zhiyuan Huang ${ }^{1}$, Rui Peng ${ }^{3}$, Jiming Peng ${ }^{1,2}$, Zuren Li ${ }^{4}$, Li Tang ${ }^{1,2}$, Ducai Liu', Xiaomao Zhou ${ }^{4}$, Lifeng Wang ${ }^{4}$, Lang Pan ${ }^{4}$, Ye Shao ${ }^{1}$, Bigang Mao ${ }^{1,2}$, Yeyun Xin ${ }^{1,2}$, Lihuang Zhu ${ }^{1,6^{*}}$, Bingran Zhao ${ }^{1,2^{*}}$ and Lianyang Bai ${ }^{1,2,4^{*}}$ (D)

\begin{abstract}
Background: Benzobicyclon (BBC) is a $\beta$-triketone herbicide $(\mathrm{bTH})$ used in rice paddy fields. It has the advantages of high efficiency, low toxicity, high crop safety, and good environmental compatibility, and shows efficacy against paddy weeds resistant to other types of herbicides. However, as some important indica rice varieties are susceptible to BBC, BBC is currently only registered and applied in japonica rice cultivation areas.

Results: By analyzing haplotypes of the bTHs broad-spectrum resistance gene HIS1 and phenotypes for BBC in 493 major indica rice accessions in China, we identified a novel non-functional allelic variant of HIS1 in addition to the previously reported 28 -bp deletion. Through detection with markers specific to the two non-functional mutations, it was clear that $25.4 \%$ of indica conventional varieties, $59.9 \%$ of fertility restorers, and $15.9 \%$ of sterile lines were susceptible to BBC. In addition, due to natural allelic variations of the HIS1 gene in the sterile and restorer lines, some two-line hybrid sterile lines were sensitive to bTHs, and the corresponding restorers were resistant. We showed the potential effectiveness of using bTHs to address the issue of two-line hybrid rice seed purity stemming from the self-crossing of sterile lines during hybrid rice seed production. Finally, allelic variations of the HIS 1 gene may also play an important role in the mechanized seed production of hybrid rice.
\end{abstract}

Conclusions: Our findings offer guidance for the application of BBC in indica rice areas and provide a nontransgenic approach to address the seed purity issue of two-line hybrid rice.

Keywords: indica rice, Hybrid rice, HIS1 gene, $\beta$-Triketone herbicides, Benzobicyclon

\section{Background}

Rice is one of the most important crops in the world (Sasaki, and International Rice Genome Sequencing, P 2005). Rice is primarily cultivated in two ways, namely

\footnotetext{
* Correspondence: Ihzhu@genetics.ac.cn; brzhao652@hhrrc.ac.cn; lybai@hunaas.cn

${ }^{\dagger}$ Qiming LV, Xiuli Zhang and Dingyang Yuan contributed equally to this work.

${ }^{1}$ State Key Laboratory of Hybrid Rice, Hunan Hybrid Rice Research Center, Changsha, China

Full list of author information is available at the end of the article
}

puddled-transplanted rice (TPR) and direct seeding of rice (DSR) (Kumar and Ladha 2011). At present, Asian countries mainly rely on TPR, while some European countries, Australia, and the United States rely almost entirely on DSR (Luo et al. 2019). Although DSR has many advantages, such as water and labor-saving potential and low methane emissions (Farooq et al. 2011; Kumar and Ladha 2011; Rao et al. 2007), it is more susceptible than TPR to weed damage. Yield losses are much higher under DSR than under TPR in the absence 
of effective weed control options (Mahajan et al. 2013; Rao et al. 2007). In countries where DSR is widely adopted, herbicide use has increased steadily; however, the long-term use of such agents can lead to the emergence of resistant weeds (Farooq et al. 2011; Gould et al. 2018; Powles and Yu 2010). Therefore, the development and application of new types of herbicides are essential for further rice production.

Benzobicyclon $(\mathrm{BBC})$ is a novel paddy-bleaching $\beta$ triketone herbicide (bTH) (Komatsubara et al. 2009) that can be applied from pre-emergence to early postemergence in paddy fields, showing broad-spectrum resistance against annual grasses, sedges, and broadleaf weeds but being harmless to TPR and DSR (Bi et al. 2018; Komatsubara et al. 2009; Maeda et al. 2019). bTHs mainly target 4-hydroxyphenylpyruvate dioxygenase (HPPD), a key enzyme in the biosynthesis of plastoquinone and tocopherol (Kraehmer et al. 2014; Moran 2005). Once the activity of HPPD is suppressed, the normal synthesis pathway of plastoquinone and tocopherol can be blocked, which in turn leads to reduced carotenoid biosynthesis and blocked electron transfer in the photosynthetic chain. Finally, photosynthesis ceases, and plants die due to bleaching (Beaudegnies et al. 2009; Kraehmer et al. 2014). As an herbicide that inhibits HPPD, BBC differs from herbicides that inhibit acetolactate synthetase (ALS) and acetyl-CoA carboxylase (ACCase), which are widely used in paddy fields (Jabusch and Tjeerdema 2005; Kumar and Ladha 2011; Mahajan et al. 2013), and thus BBC can be used as a rotation herbicide in these fields. The number of weed species with resistance to herbicides that inhibit HPPD is minimal (Gould et al. 2018), and BBC shows efficacy against paddy weeds resistant to other types of herbicide, including sulfonylureas (Maeda et al. 2019).

$\mathrm{BBC}$ is a selective herbicide. To date, it has only been registered and applied in japonica rice cultivation areas because some indica rice varieties are susceptible to BBC. Recently, the rice gene HIS1 (HPPD INHIBITOR SENSITIVE 1), which confers resistance to $\mathrm{BBC}$ and other bTHs in japonica varieties, was identified. HIS1 encodes a $\mathrm{Fe}$ (II)/2-oxoglutarate (2OG)-dependent oxygenase that detoxifies bTHs by catalyzing their hydroxylation (Maeda et al. 2019). Sequence alignment revealed that his1 in BBC-sensitive varieties harbors a 28-bp deletion in the third exon, which leads to a nonsense mutation (Maeda et al. 2019). PCR-based genotyping showed that the 28-bp deletion was not found in most tested japonica rice varieties but was present in some indica rice varieties, including Tadukan and IR64, which are backbone parents used in indica rice breeding (Huang et al. 2018; Mackill and Khush 2018). Therefore, it can be inferred that many modern indica rice varieties are susceptible to $\mathrm{BBC}$ due to the nonfunctional his1 with the 28-bp deletion.

China is one of the main rice-producing countries, with an annual rice cultivation area that exceeds 30 million $\mathrm{hm}^{2}$ (Hu et al. 2016). China's rice cultivation area can be broadly divided into northern japonica rice areas and southern indica rice areas. The area planted with indica rice in China accounts for more than $70 \%$ of the total rice area (Wan et al. 2010; Zhang et al. 2016). In view of the excellent performance of $\mathrm{BBC}$ in weed control, clarifying which indica rice varieties can tolerate this type of herbicide should offer many benefits. The vast majority of hybrid rice varieties are indica, and the annual hybrid rice cultivation area exceeds 16.7 million $\mathrm{hm}^{2}$ in China due to good yield performance (Hu et al. 2016). In addition, the cultivation area of hybrid rice has also continued to increase in other countries (Xie and Peng 2016). However, although hybrid rice has many advantages, its seed production process is complicated. Hybrid rice parents need to be planted separately, and hybrids and male parents need to be harvested separately, which is time-consuming, laborious, and not suitable for mechanization (Xia et al. 2019). By breeding restorer lines containing herbicide-sensitive genes, the herbicides can be sprayed in the seed production field immediately after pollination, and the male parent can be selectively killed, thereby realizing hybrid rice mixedsowing and mechanized harvesting. In addition, in the two-line hybrid rice seed production process, the fertility of photoperiod-and thermo-sensitive genic male sterile (P/TGMS) lines is susceptible to temperature, causing the P/TGMS lines to self-cross, thus affecting the purity of the hybrid seed (Ding et al. 2012; Zhou et al. 2012; Zhou et al. 2014). When the purity of the two-line hybrid seed is not sufficient, these hybrid seeds cannot be used in production and will cause significant economic losses. For example, about 6700 ha of two-line hybrid rice failed to produce seed due to the continued low temperature in Jiangsu, Anhui, and Sichuan provinces in China in 2009, which caused a direct economic loss of nearly 100 million Yuan. Furthermore, due to the shortage of hybrid rice seeds, the cultivation area of two-line hybrid rice was reduced by 1.3 million hectares in 2010 (Chen et al. 2011). Through the breeding of P/TGMS lines containing herbicide-sensitive genes, the application of herbicides to hybrid paddy fields at the seedling stage can selectively kill the P/TGMS lines to achieve hybrid purification. There have been many attempts to use herbicides in hybrid rice seed production and hybrid purification, and these attempts have mainly relied on mutagenic and transgenic approaches (Komatsubara et al. 2009; Pan et al. 2006; Shoba et al. 2017; Xia et al. 2019; Zhang et al. 2002; Zhang et al. 2014). However, mutagenesis can only create a few specific materials, and 
its application is limited. Transgenic rice is not commercially planted in China. The direct selection of materials with different responses to herbicides among the existing hybrid rice parents is likely to play an important role in the further development of hybrid rice.

The aim of this work was to evaluate the potential applications of bTHs in indica rice. We performed haplotype analysis of the HIS1 gene in more than 600 indica rice accessions, including hybrid rice parents commonly used in China. In addition to the previously reported 28bp deletion, we also discovered a novel mutation that caused a loss of function of the HIS1 gene, and we further clarified which indica rice varieties can tolerate bTHs through specific marker detection. We also provided potential applications of bTHs for indica cultivation areas and hybrid rice seed production and purification.

\section{Results}

\section{Detection of the 28-Bp Deletion and Phenotyping of indica}

Based on the reported 28-bp deletion that leads to different reactions to $\mathrm{BBC}$ among rice cultivars (Maeda et al. 2019), an InDel marker was developed to detect this variation in 631 indica accessions commonly used in rice breeding in China (Table S1, Figure S1). The results showed that the 28-bp deletion was widely distributed among China's indica accessions, with an average rate of $28.1 \%$. Among them, the deletion rate of the 3line restorer was the highest, reaching $50.7 \%$, whereas no deletion was found in the 87 3-line cytoplasm male sterile (CMS) lines. Forty (18.1\%) conventional lines, 24 (40.7\%) 2-line restorers, and five (9.8\%) 2-line genic male sterile (P/TGMS) lines harbored the 28-bp deletion (Table 1). In general, there were more than 28-bp deletions in the restorer lines (48.5\%), followed by conventional rice and then the sterile lines (3.6\%).

In order to determine whether the $\mathrm{BBC}$-sensitive phenotype is completely related to the 28-bp deletion, we selected 493 accessions including conventional and restorer lines (sterile line seeds are more valuable, and we did not have enough seeds) for BBC application. At first, Nipponbare (NB) (HIS1) and IR64 (his1) were used to determine suitable $\mathrm{BBC}$ concentrations. The results showed that IR64 started to show signs of injury when the concentration was $0.03 \mathrm{~g} / \mathrm{L}$. When the concentration reached $0.15 \mathrm{~g} / \mathrm{L}$, the difference between IR64 and NB was already obvious, and when the concentration reached $1.2 \mathrm{~g} / \mathrm{L}, \mathrm{NB}$ also started to show susceptibility (Fig. 1a, b). Ultimately, a concentration of $0.4 \mathrm{~g} / \mathrm{L}$ was selected to evaluate the resistance of indica accessions to $\mathrm{BBC}$. According to the different responses of rice to $\mathrm{BBC}$, we divided the phenotypes into six grades: grades 0 and 1 showed complete resistance, grades 2 and 3 were intermediate types, and grades 4 and 5 were completely sensitive (Fig. 1c). The BBC spraying results showed that most indica accessions showed high resistance or high sensitivity, and only a few were of the intermediate type, which is probably related to the HIS1 gene as the major BBC resistance gene (Maeda et al. 2019) (Fig. 1d). Among the three different types of rice accessions, the $\mathrm{BBC}$-sensitive trait had a certain relationship with the 28-bp deletion, but the number of $\mathrm{BBC}$ sensitive accessions obviously exceeded the number of 28-bp deletions (Table 1). Therefore, in addition to the 28-bp deletion, there may be other mutation(s) of the HIS1 gene that lead to BBC sensitivity.

\section{The T1510G Mutation in the HIS1 Gene Leads to the Loss of Function}

In order to determine whether BBC sensitivity is caused by mutations of the HIS1 gene, we performed PCR cloning and sequencing of the HIS1 gene in 493 phenotyped indica accessions. The sequencing results showed that there were only five mutation sites in the exons of HIS1, which divided the HIS1 gene into five haplotypes (Fig. 2a), among which $\mathrm{H} 2$ and $\mathrm{H} 5$ are alleles of the HIS1 gene in Nipponbare and IR64, respectively. Compared with H2, H1 had two mutations (C9T, G1055A), H3 had only one mutation (T1510G), H4 had two mutations (G61C, G1055A), and the 28-bp deletion was found only in $\mathrm{H} 5$. When the phenotype of each indica accession was correlated with the haplotype of the HIS1 gene, we found that accessions containing $\mathrm{H} 1, \mathrm{H} 2$, and

Table 1 Indica accession numbers of the 28-bp deletion, T1510G mutation, and lines susceptible to BBC

\begin{tabular}{llllll}
\hline & Total & 28-bp deletion & HS & MS & T1510G \\
\hline Conventional varieties & 221 & $40(18.1 \%)$ & $55(24.9 \%)$ & $1(0.5 \%)$ & $16(7.2 \%)$ \\
3-line restorer & 213 & $108(50.7 \%)$ & $129(60.6 \%)$ & $3(1.4 \%)$ & $24(11.3 \%)$ \\
2-line restorer & 59 & $24(40.7 \%)$ & $29(49.2 \%)$ & $2(3.4 \%)$ & $7(11.9 \%)$ \\
CMS & 87 & 0 & NA & NA & $14(16.1 \%)$ \\
P/TGMS & 51 & $5(9.8 \%)$ & NA & NA & $3(5.9 \%)$ \\
Total & 631 & $177(28.1 \%)$ & NA & $64(10.1 \%)$ \\
\hline
\end{tabular}

CMS 3-line cytoplasm male sterility, P/TGMS Photoperiod- and thermo-sensitive genic male sterile, HS Highly susceptible, MS Moderately susceptible, NA No phenotype 

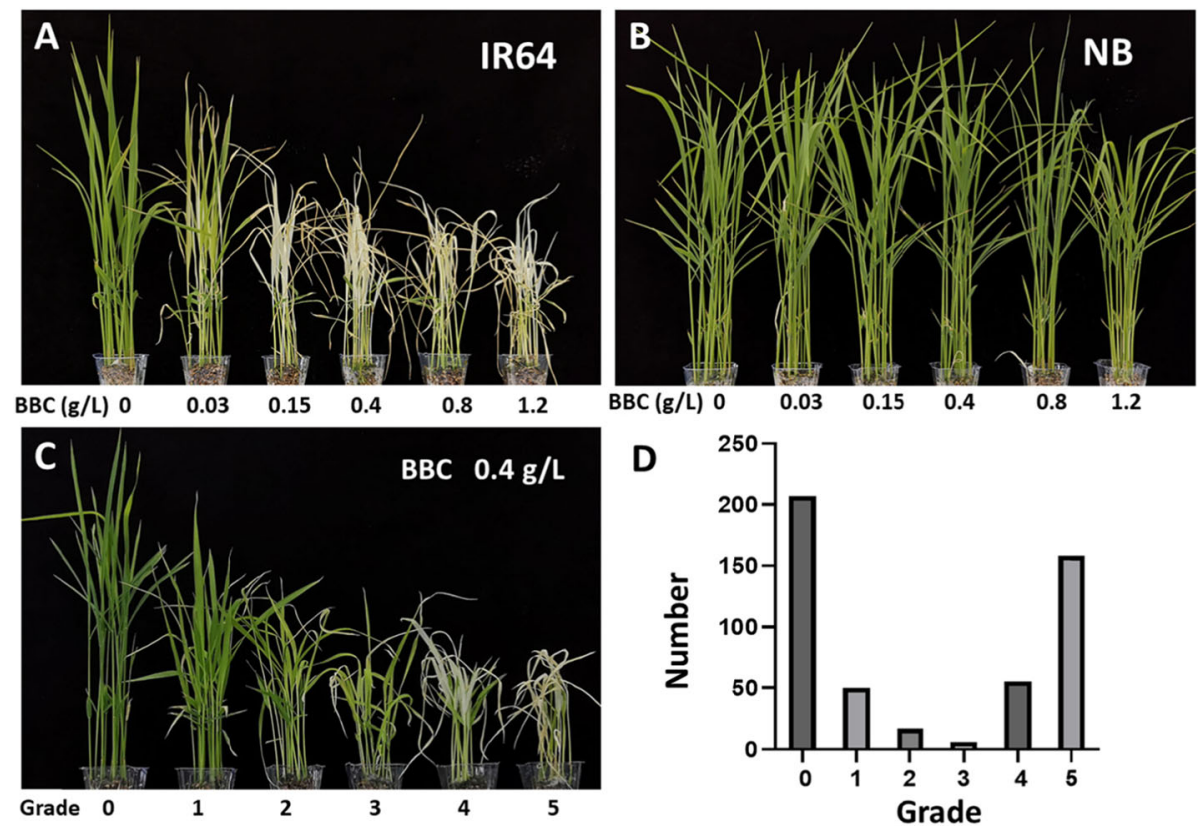

Fig. 1 Phenotyping of indica rice accessions. a Concentration-dependent effects of BBC on IR64. b Concentration-dependent effects of BBC on Nipponbare (NB). c Different graded phenotypes after treatment with $0.4 \mathrm{~g} / \mathrm{L}$ BBC. $\mathbf{d}$ Number of accessions with different graded phenotypes. Grade 0: unaffected seedling; grade 1: seedling growth was slightly weakened; grade 2: seedlings with an obvious dwarfing phenomenon; grade 3: seedlings without obvious bleaching but where some had started to die; grade 4: seedlings bleached completely; grade 5: seedlings completely died

$\mathrm{H} 4$ showed full resistance to $\mathrm{BBC}$, while $\mathrm{H} 3$ and $\mathrm{H} 5$ showed complete susceptibility to BBC (Fig. 2a). Studies have shown that non-functional H5 is related to the 28bp deletion (Maeda et al. 2019). As H3 had only one single base mutation compared with functional $\mathrm{H} 2$, it was initially believed that this T1510G mutation was the cause of the loss of function.

The T1510G mutation in H3 caused an amino acid change (V286G) at the protein level (Figure S2), located in the region of the putative substrate pocket that is very important for the function of Fe (II)/2OG-dependent oxygenases (2OGD) (Maeda et al. 2019) (Fig. 2b). After amino acid sequence alignment of 91 experimentally characterized 2OGDs in plants and 114 putative 2OGDs in rice (Kawai et al. 2014), respectively, it was found that this site located in the most conservative motif, and amino acids $\mathrm{Val}$ and Leu were dominant at this site (Figure S3). In order to exclude the possibility that nonfunctional $\mathrm{H} 3$ was due to different expressions of HIS1, we randomly selected five accessions harboring $\mathrm{H} 2$ and $\mathrm{H} 3$ to detect the HIS1 mRNA level, using NB and IR64 as controls. The results showed that HIS1 was normally expressed in all accessions with $\mathrm{H} 3$, and there was no obvious correlation between HIS1 gene expression and anti-susceptibility to BBC (Fig. 2c). Moreover, through qRT-PCR detection, it was also found that there was no significant difference in the expression levels of each
HIS1 haplotype (Figure S4). An F2 population of Nongxiang42 (containing H2) and Xianhui207 (containing H3) was constructed to analyze the relationship between susceptibility to BBC and the T1510G mutation. The results of spraying $\mathrm{BBC}$ on the F2 population showed that among the 968 individual plants, 737 individual plants showed resistance, and 231 individual plants showed sensitivity to BBC. The ratio of resistant and sensitive plants showed a 3:1 separation ratio $\left(X^{2}=0.607<\right.$ $\left.\mathrm{X}_{(0.05)}{ }^{2}=3.84\right)$, which demonstrated that there was only one dominant resistance gene in Nongxiang42. Next, we designed a dCAPS marker based on the T1510G mutation to distinguish $\mathrm{H} 3$ from other haplotypes (Fig. 2d, e). Using this H3-specific marker to detect individual plants of the F2 population, we found that the T1510G mutation was completely co-segregated with susceptibility to BBC (Figure S5). In summary, our results showed that the T1510G mutation caused the loss of function of the HIS1 gene.

\section{Natural Allelic Variations of HIS1 in Cultivated Rice}

In order to fully evaluate which indica rice accessions can tolerate BBC, we tested all P/TGMS and CMS lines with the H3-specific marker. The results showed that there were three P/TGMS and 14 CMS lines that harbored the T1510G mutation (Table 1). By combining the detection results of the 28-bp deletion in the early stage, 


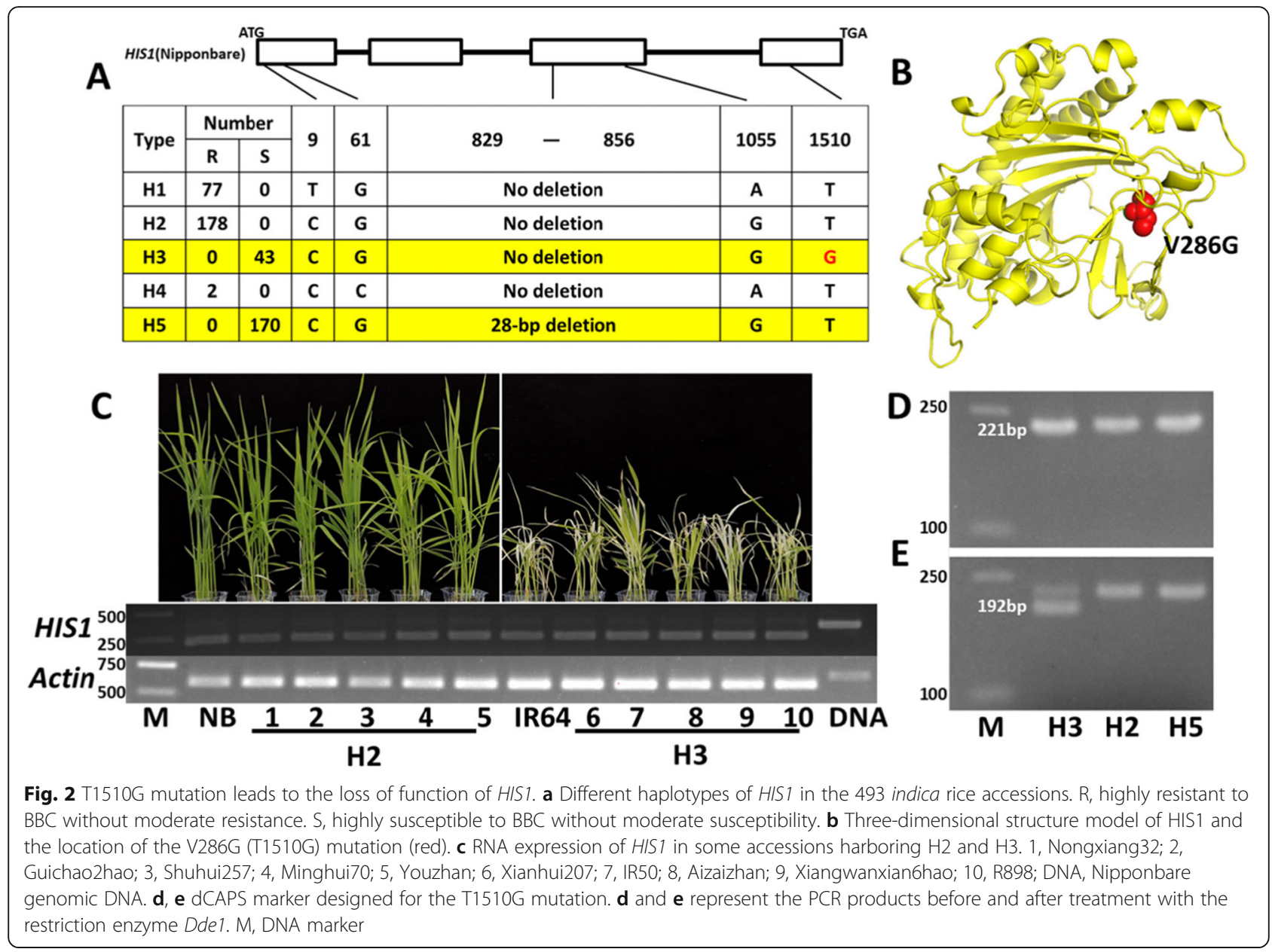

we concluded that about $40 \%$ of the indica rice materials and about $25 \%$ of conventional rice should be sensitive to BBC (Table 1). Although the proportion of 3- and 2line restorers that are sensitive to $\mathrm{BBC}$ was very high (60\% and $52.6 \%$, respectively) due to the low proportion of P/TGMS and CMS lines, the ratio of the indica hybrids that are sensitive to $\mathrm{BBC}$ should be very low, considering that HIS1 is a major dominant resistance gene.

In order to clarify the distribution of non-functional HIS1 alleles in cultivated rice, we used the 3000 sequenced rice genomes (Wang et al. 2018) to conduct a comprehensive analysis of the 28-bp deletion and T1510G mutation of the HIS1 gene. The results showed that among 1716 indica rice accessions, 117 harbored the 28-bp deletion, and 292 accessions contained the T1510G mutation; among 825 japonica rice accessions, no 28-bp deletion was found, and only three accessions had the T1510G mutation. Non-functional alleles of the HIS1 gene are rarely found in japonica rice, and this phenomenon could explain why the BBC herbicide is registered and can be used safely in the japonica rice area. In the two other types of cultivated rice, aus and aro, there was no 28-bp deletion, and only one T1510G mutation was found in aus (Fig. 3a). Unlike the 3000 rice accessions, which are mainly landraces with wide genetic variations (Wang et al. 2018), the 631 indica accessions we tested were mainly Chinese indica rice breeding materials. Non-functional alleles of HIS1 increased significantly in our 631 indica accessions, especially in the restorer lines (Fig. 3b), compared with the 3000 rice accessions, which is likely the result of artificial selection during the rice breeding process.

\section{BBC Is Applicable for Two-Line Hybrid Rice Seed Purification}

In order to evaluate the possibility of using the HIS1 gene to address 2-line hybrid rice seed purity issues, we sprayed $\mathrm{BBC}$ on the eight $\mathrm{P} / \mathrm{TGMS}$ lines that harbored the 28-bp deletion or the T1510G mutation. The results showed that all eight $\mathrm{P} / \mathrm{TGMS}$ lines were susceptible to BBC (Fig. 4a). In addition, a BBC concentration gradient experiment was conducted on P/TGMS lines SE21S (T1510G) and MingS (28-bp deletion) with Y58S as a control. The results showed that SE21S and MingS were injured at a low BBC concentration $(0.05 \mathrm{~g} / \mathrm{L})$, while Y58S exhibited no obvious damage until the $\mathrm{BBC}$ 

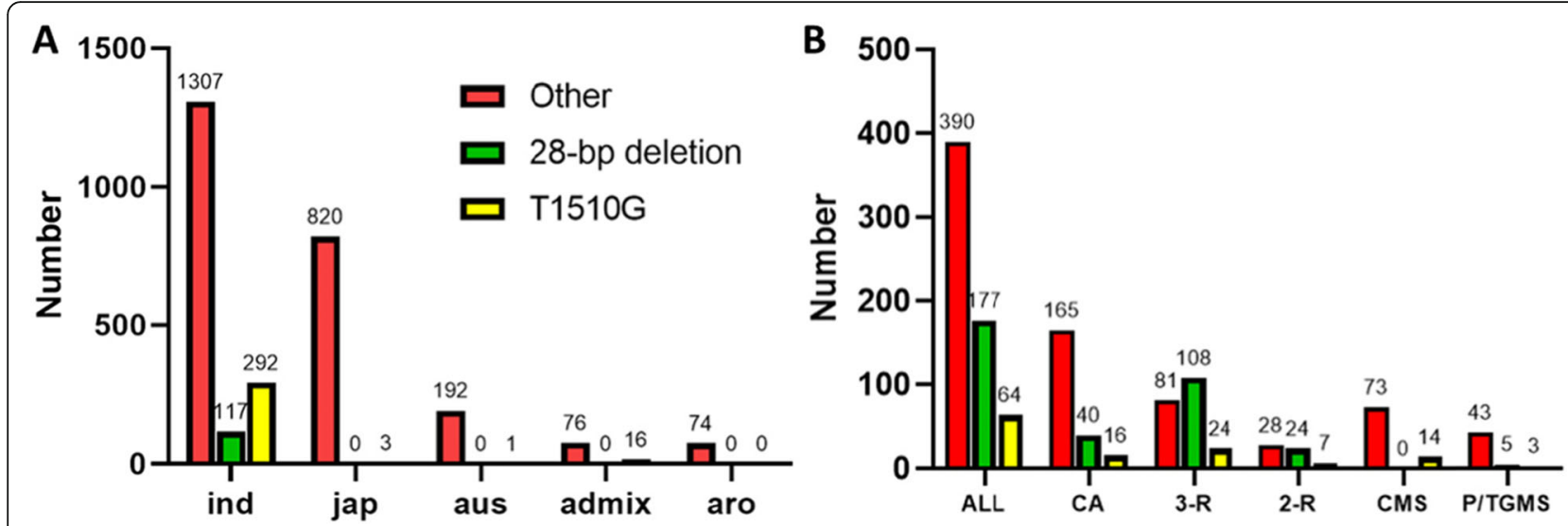

Fig. 3 Comparison of the non-functional alleles of HIS1 in different rice accessions. a Numbers of non-functional alleles of HIS1 in 3000 rice accessions. Ind, indica; jap, japonica; aus, aus accessions; aro, aromatic accessions; admix, all other unassigned accessions. b Numbers of nonfunctional alleles of HISI in indica rice accessions collected in this study. ALL, all indica accessions; CA, conventional accessions; 3-R, 3-line restorer; 2-R, 2-line restorer; CMS, 3-line cytoplasm male sterile line; P/TGMS, photoperiod- and thermo-sensitive genic male sterile

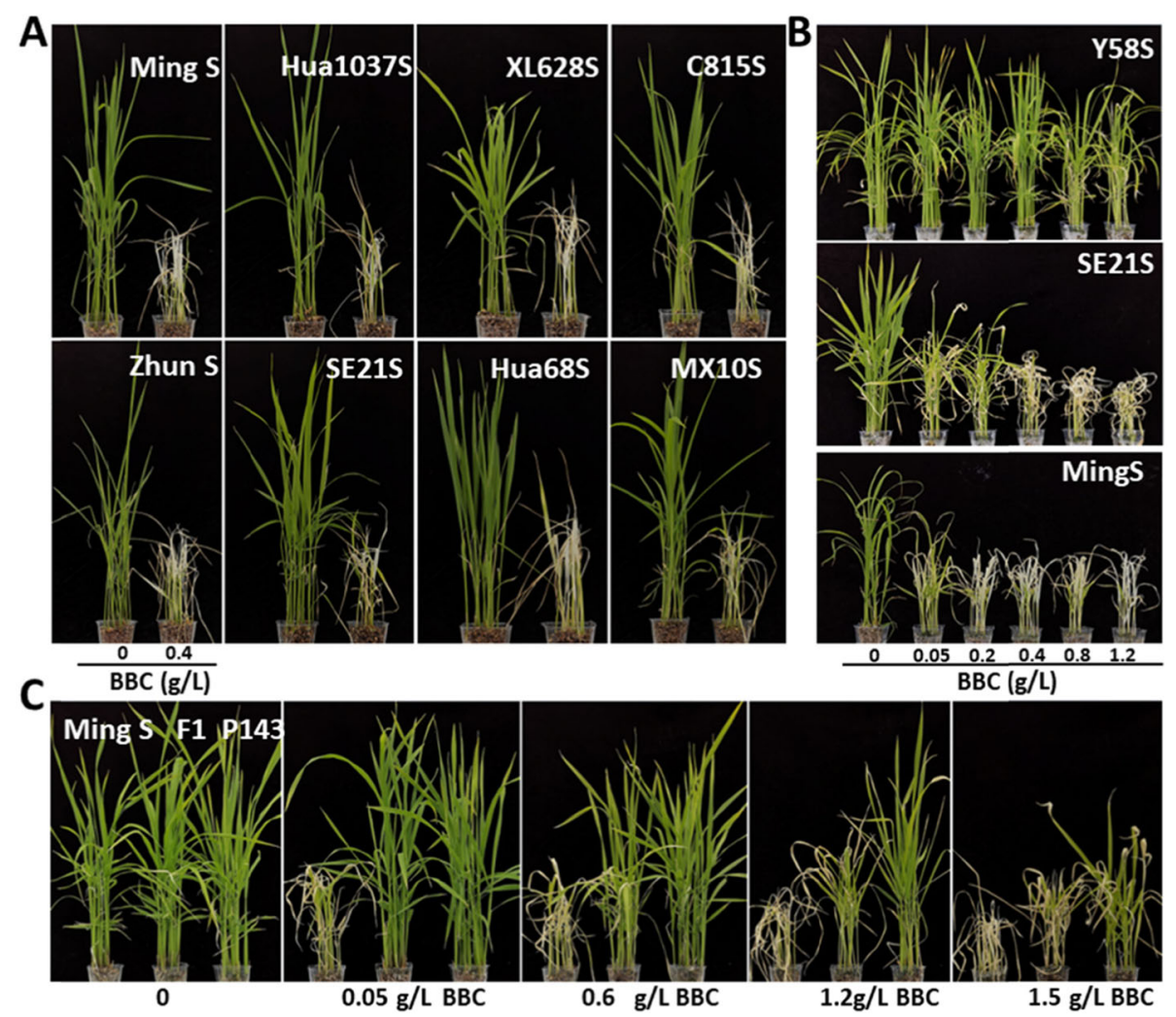

Fig. 4 Potential applications of BBC in improving the seed purity of 2-line hybrid rice. $\mathbf{a}$ Some 2-line P/TGMS are susceptible to BBC. $\mathbf{b}$ Concentration-dependent effects of BBC on three representative 2-line P/TGMS accessions, Y58S (HIS1), SE21S (his1-T1510G), and MingS (his1-28bp deletion). c Concentration-dependent effects of BBC on the 2-line hybrid variety Mingliangyou143 (F1), female parent MingS, and male parent P143 
concentration reached $1.2 \mathrm{~g} / \mathrm{L}$ (Fig. 4b). Finally, we chose a commercialized 2-line hybrid rice variety Mingliangyou143 from China, of which the female and male parents are MingS and P143 (containing HIS1 H2), respectively, to examine the effect of $\mathrm{BBC}$ on the purification of 2-line hybrid rice seeds. The results showed that at least in the range of $0.05-0.6 \mathrm{~g} / \mathrm{L}, \mathrm{BBC}$ was very effective in killing the female parent MingS but was harmless to the Mingliangyou143 hybrid (Fig. 4 C). Moreover, it was found that BBC could kill the P/TGMS line without affecting the hybrid when a small amount of MingS was mixed into Mingliangyou143 (Figure S6). At high concentrations, the hybrid containing the heterozygous HIS1/his1 gene appeared to be less resistant to $\mathrm{BBC}$ than the homozygous male parent, and there were sufficient $\mathrm{BBC}$ concentration intervals for the purification of the 2-line hybrid seeds.

To determine whether BBC could be used to address 2-line hybrid rice seed purity issues in the field, we sprayed BBC on Mingliangyou143 and its parents in the field. We found that under direct seeding cultivation, when the $\mathrm{BBC}$ dosage was $300 \mathrm{~g} / \mathrm{ha}$ (the recommended dosage is $150-225 \mathrm{~g} / \mathrm{ha}$ for weeding in the field), all seedlings of the female parent MingS showed obvious bleaching, and growth stopped completely. By contrast, Mingliangyou143 and the male parent P143 grew normally (Fig. 5). These results demonstrated that BBC could address the problem of 2-line hybrid rice seed purity during weed control in the field.

\section{Potential Applications of Other $\beta$-Triketone Herbicides}

The commonly used bTHs include BBC, mesotrione, sulcotrione, tembotrione, and tefuryltrione. Currently, mesotrione is the most widely used HPPD-inhibitor herbicide globally (Hua 2015). In order to identify whether other bTHs are the same as BBC, i.e., can be used in indica rice areas and 2-line hybrid rice seed purification, we chose mesotrione for further study. As shown in Fig. 6a-d, IR64 and SE21S started to become susceptible to mesotrione when the concentration exceeded $0.01 \mathrm{~g} / \mathrm{L}$. However, NB and Y58S only started to show obvious injury when the mesotrione concentration reached $0.45 \mathrm{~g} / \mathrm{L}$. In addition, the spraying of a mesotrione concentration gradient on Mingliangyou143 and its parents showed that mesotrione could also be used for the purification of 2-line hybrid rice seed, at least when the application concentration ranges from $0.01 \mathrm{~g} / \mathrm{L}$ to $0.1 \mathrm{~g} / \mathrm{L}$ (Fig. 6e). Since the HIS1 gene has been confirmed to be a broad-spectrum resistance gene for bTHs (Maeda et al. 2019) and mesotrione is similar to $\mathrm{BBC}$ in terms of the phytotoxicity responses of different indica rice varieties, we determined that by detecting the HIS1 gene in indica rice, there is potential to use bTHs in indica rice areas and in the purification of 2line hybrid rice seeds.

\section{Discussion}

Potential Applications of BBC in indica Rice Areas

bTHs are HPPD inhibitors that are applied widely in crops (Kraehmer et al. 2014). These inhibitors exhibit various advantages, including their high efficiency, low toxicity, safe application in crops, effectivity against resistant weeds, low environmental impact, and low impacts on subsequent crops. As a bTH herbicide used in paddy fields, BBC is currently only registered and applied in japonica rice cultivation areas. Through analysis of the non-functional mutations of the HIS1 gene in 3000 rice accessions, we found that there was almost no non-functional HIS1 allele in japonica rice; this is consistent with the fact that $\mathrm{BBC}$ can be registered and applied in japonica rice areas. In addition, in the aus and aro rice accessions, the ratio of non-functional his1 was

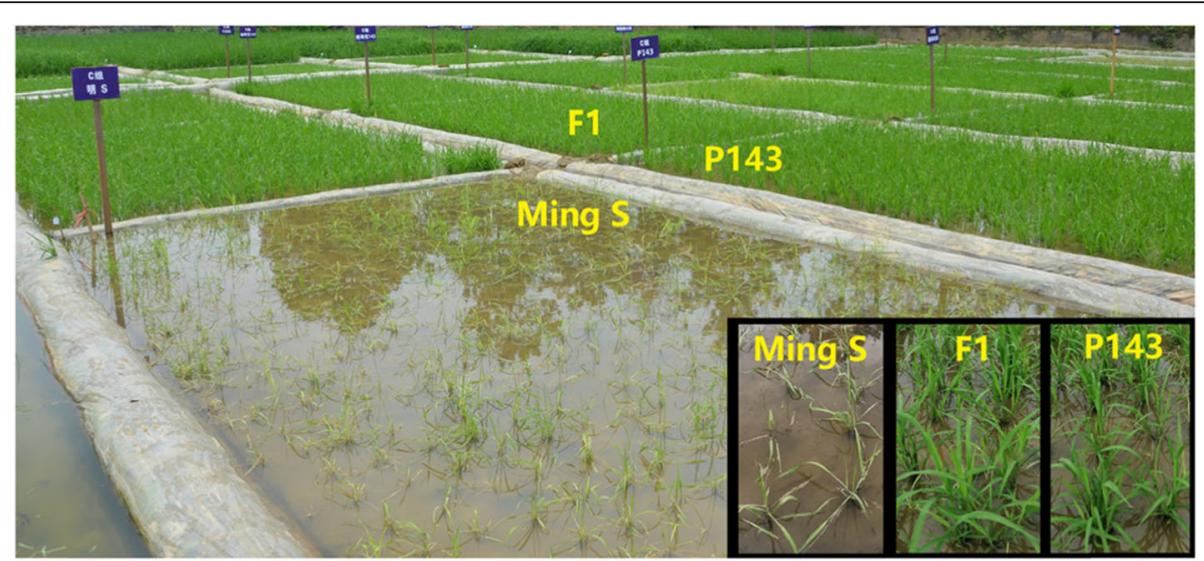

Fig. 5 Field phenotype of Mingliangyou143 and its parents to BBC. The 2-line hybrid rice variety Mingliangyou143 (F1) and its female parent MingS and male parent P143 were grown in a paddy field treated with BBC ( $300 \mathrm{~g}$ of active ingredient/ha) at 10 days after direct seeding. Examination of the plants at 25 days after direct seeding revealed that F1 and P143 were resistant to BBC, whereas MingS was sensitive 


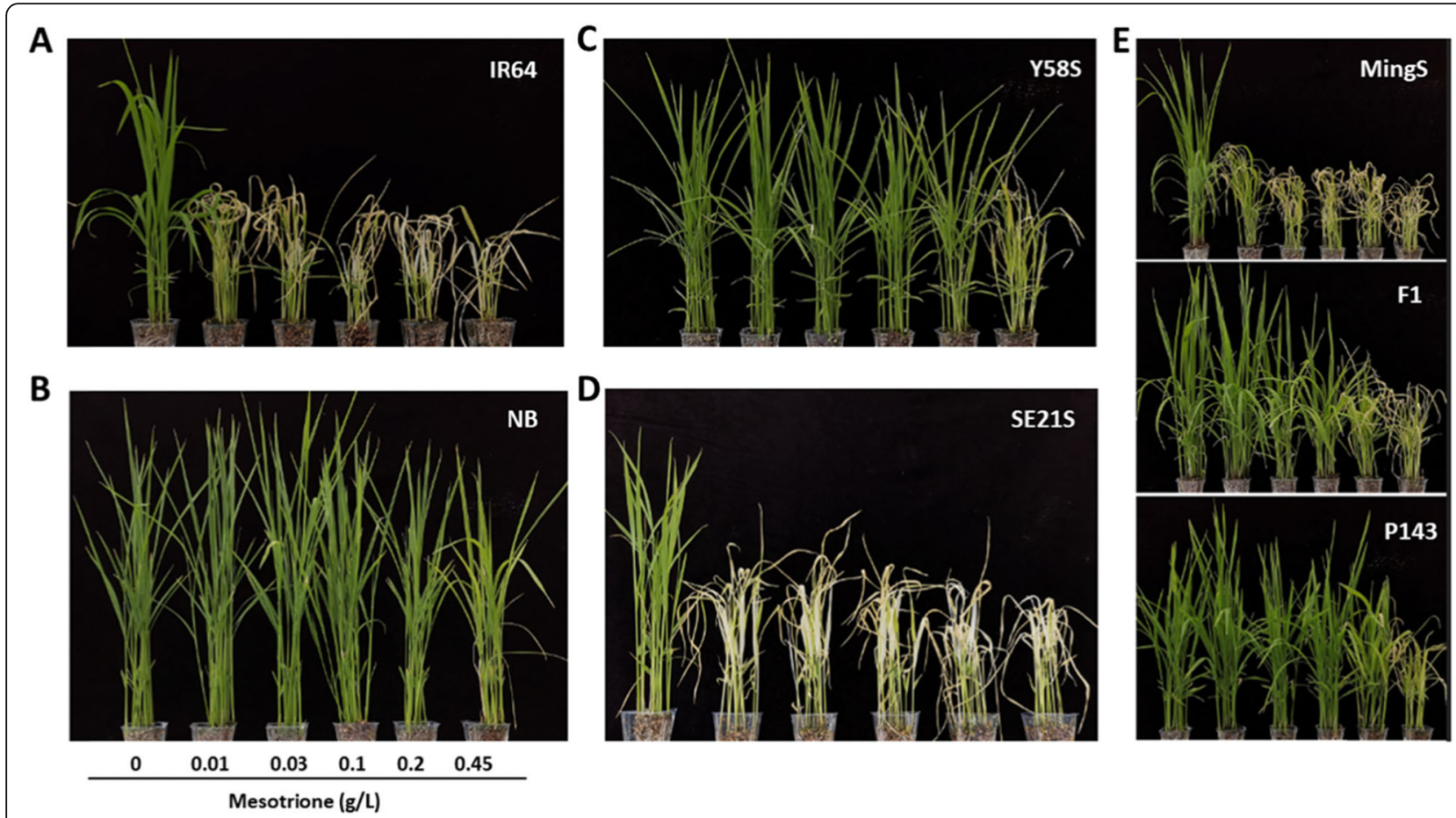

Fig. 6 Potential applications of mesotrione in improving the seed purity of 2-line hybrid rice. a-d Concentration-dependent effects of mesotrione on IR64, Nipponbare (NB), Y58S, and SE21S. e Concentration-dependent effects of mesotrione on the 2-line hybrid variety Mingliangyou143 (F1), female parent MingS, and male parent P143

also very low, and thus the application of $\mathrm{BBC}$ may be potentially promising in these two types of rice cultivation areas. In cases where some important indica rice varieties are $\mathrm{BBC}$-sensitive, it is important to determine the application scope of $\mathrm{BBC}$ in indica rice areas. This would allow BBC to be applied to a large area, which could resolve the issue of the resistance of weeds to other types of herbicide, including sulfonylureas (Maeda et al. 2019). In this study, by sequencing the bTHs broad-spectrum resistance gene HIS1 in 493 indica rice accessions commonly used as conventional lines and restorers of hybrid rice in China, a total of five haplotypes of HIS1 were identified. Among them, $\mathrm{H} 1, \mathrm{H} 2$, and $\mathrm{H} 4$ were resistant to $\mathrm{BBC}$, while $\mathrm{H} 3$ and $\mathrm{H} 5$ were nonfunctional. The loss of function of $\mathrm{H} 5$ was due to the previously reported 28-bp deletion in the third exon, while the non-functional H3 was found to be caused by the T1510G mutation in the fourth exon in this study. Two markers specific to the 28-bp deletion and the T1510G mutation were designed to test the indica rice accessions. Ultimately, we determined that approximately $25 \%$ of conventional rice, $15 \%$ of the sterile lines, and $60 \%$ of the restorer lines harbored the two nonfunctional mutations in HIS1. Considering that the HIS1 gene is a major dominant resistance gene, hybrid rice varieties containing heterozygous HIS1/his1 could be resistant to bTHs. Therefore, although the ratio of non- functional HIS1 alleles was much higher in the restorer lines compared to the other types of rice, the ratio of hybrid rice varieties susceptible to bTHs should be relatively low. In addition, through concentration gradient experiments, it was found that there was no particularly significant difference between resistant indica and japonica rice. Therefore, as rice cultivation in China has largely begun to be transformed into agricultural cooperatives and large grain farms, it would be possible to apply $\mathrm{BBC}$ herbicides in specific indica rice areas in the future.

\section{Potential Applications of bTHs in Seed Purification of 2- Line indica Hybrid Rice}

At present, indica rice areas in China are mainly planted with hybrid rice, with an annual planting area of 16 million $\mathrm{hm}^{2}$, of which 2-line hybrid rice accounts for more than $30 \%$ (Hu et al. 2016). In addition, in other countries, such as the United States, 2-line hybrid rice is also being planted on a large scale (Xie and Peng 2016). Although 2-line hybrid rice has the advantages of simpler breeding procedures and more freedom in combination, the P/TGMS sterile lines are negatively affected by light and temperature during seed production, resulting in self-crossing, which seriously affects the quality of hybrid seeds. Previous studies have attempted to create herbicide-sensitive sterile lines or herbicide-resistant 
restorer lines through genetic modification and physical/ chemical mutagenesis (Shoba et al. 2017; Tang et al. 2020; Xia et al. 2019; Zhang et al. 2002; Zhang et al. 2014), but have not resolved the seed purity problem of 2-line hybrid rice at a large scale except for the use of imidazolinone-resistant restorers in the United States (Sudianto et al. 2013). In this study, we identified eight 2-line sterile lines naturally sensitive to bTHs, whereas many of the corresponding restorer lines were resistant. Therefore, in this two-line hybrid rice, bTHs can be used directly to purify hybrid rice seeds. Moreover, we found that mesotrione required a lower lethal dose and had lower costs than BBC for seed purification. Since the HIS1 gene has no other side effects on rice growth and yield (Maeda et al. 2019), it would be possible to introduce his1 into 2-line sterile lines and HIS1 into restorer lines in future research to control against the risks of 2line hybrid rice seed purity.

\section{Potential Applications of bTHs in the Mechanized Seed Production of indica Hybrid Rice}

By comparing the ratio of non-functional HIS1 alleles in the indica accessions of the 3000 rice accessions and in commonly used indica rice in China, we found that the percentage of non-functional alleles of the HISI gene in breeding materials in China, especially in the restorer lines, was much higher. By analyzing each material, we found that this increased percentage might be due to some important backbone restorer lines, such as IR24, IR26, Minghui63, Shuhui527, and Mianhui725 (supplementary Table 1), all of which contained the nonfunctional alleles of HIS1. Considering there are many bTH-sensitive restorer lines and bTH-resistant sterile lines, mixed seeding and mechanized harvesting are feasible in the seed production of some hybrid rice varieties, as bTHs can be sprayed after pollination to kill the male parent, leaving only real hybrids. In addition, it is possible that the use of bTHs causes the incomplete killing of restorers after pollination (Tang et al. 2020). This method can therefore also be combined with other hybrid rice mechanized seed production methods, such as the use of husk color differences, grain size differences, or female sterile material systems (Tang et al. 2020; Xia et al. 2019).

\section{Conclusions}

Both the T1510G mutation and the previously reported 28-bp deletion caused the loss of function of the HIS1 gene. Approximately $75 \%$ of conventional indica rice varieties and most indica hybrid rice varieties in China are able to tolerate bTHs. Allelic variations of the HIS1 gene in hybrid rice parents provide a non-transgenic approach that can be widely applied to address the seed purity issue of 2-line hybrid rice.

\section{Methods}

\section{Plant Materials and Genotyping}

A total of 631 indica rice accessions commonly used in Chinese rice breeding were used in this study, including 221 conventional lines, 213 hybrid rice 3-line restorers, 59 hybrid rice 2-line restorers, $87 \mathrm{CMS}$, and 51 P/TGMS (supplementary Table 1). Details of these rice accessions are listed in the "China Rice Data Center" (http://www. ricedata.cn/variety/index.htm). Indica IR64 (BBC-sensitive) and japonica Nipponbare (BBC-resistant) were used as controls. An F2 population derived from a cross between Nongxiang42 (BBC resistant with HIS1 H2) and Xianhui207 (BBC sensitive with HIS1 H3) was used to test the relationship of the T1510G mutation and the susceptibility to BBC. Genomic DNA fragments from the start codon to the stop codon of the HIS1 gene were obtained by high-fidelity PCR. The PCR products were directly sequenced by TsingKe Biotech (Changsha, China). Genotyping with an InDel marker specific to the 28-bp deletion was performed as previously described (Maeda et al. 2019). A dCAPS marker specific to the T1510G mutation in the HIS1 gene was designed with the online software dCAPS Finder 2.0 (http://helix.wustl. edu/dcaps/dcaps.html), and the restriction enzyme Dde1 was obtained from New England Biolabs (PCR primers are listed in Table S2). Genotypes of the HIS1 gene in 3000 rice accessions and the classification information of these rice accessions were obtained from the Rice SNPSeek Database (Alexandrov et al. 2015) (https://snp-seek. irri.org).

\section{Chemicals}

The herbicide benzobicyclon (BBC) (trade name "Cong Feng", 25\% active ingredients) was obtained from SDS Biotech (Tokyo, Japan). The herbicide mesotrione (trade name "Qian Xiao", 10\% active ingredients) was obtained from FengShan Corp. (Jiangsu, China).

\section{Detection of the Susceptibility of Rice Accessions to Herbicide}

All procedures were carried out as described in a previous study (Maeda et al. 2019), with some modifications: 493 rice seeds were soaked for 2 days and germinated for 1 day at $37^{\circ} \mathrm{C}$. About $50 \mathrm{~mL}$ of vermiculite was mixed with half-strength MS culture medium (no sugar and agar) (obtained from BaiSi Biotech, Hangzhou, China) and placed in a plastic cup, and the germinated rice seeds were placed on the surface of the vermiculite. Seedlings were grown at $28^{\circ} \mathrm{C}$ for $8 \mathrm{~d}$ with $16 \mathrm{~h}$ of light and $8 \mathrm{~h}$ of dark per day. After the first leaves had expanded, water or water containing herbicide was sprayed on the leaves of the rice seedlings. Seedling growth was assessed after 10 or $15 \mathrm{~d}$. Each rice accession was tested three times for sensitivity to $\mathrm{BBC}$ or mesotrione. 
Compared with the control, unaffected seedling growth was designated as grade 0 ; seedling growth that was slightly weakened was designated as grade 1 ; seedlings with an obvious dwarfing phenomenon were designated as grade 2; seedlings without obvious bleaching but that had started to die were designated as grade 3; seedlings that had bleached completely were designated as grade 4; and seedlings that died were designated as grade 5 . Grades 0 and 1 were designated as completely resistant; grades 2 and 3 were designated as moderately resistant and moderately susceptible, respectively; and grades 4 and 5 were designated as completely susceptible. The 2line hybrid rice variety Mingliangyou143 and its female parent MingS and male parent P143 were planted by direct seeding in a paddy field. After the second leaves had expanded ( $10 \mathrm{~d}$ after seeding), BBC ( $300 \mathrm{~g}$ of active ingredient/ha) was sprayed on the leaves of the rice seedlings. Seedling growth was assessed after $15 \mathrm{~d}$.

\section{Expression Profile of HIS1 Genes}

Total RNA was extracted from rice seedlings using an RNeasy Plant Mini Kit (Qiagen). Semi-quantitative PCR was conducted as previously described (Maeda et al. 2019) with the rice actin gene as an internal control. PCR amplification of the HIS1 gene was 35 cycles, and that of the actin gene was 28 cycles. Quantitative PCR analysis was performed with the TransScript Green One-Step qRT-PCR System (TransGen Biotech). The specific primers CCJQ-F and CCJQ-R were used for detection of the HIS1 and ActinQ-F and ActinQ-R for the reference gene actin.

\section{Alignment of Protein Sequences}

Amino acid sequence alignment of 91 experimentally characterized 2OGDs in plants and 114 putative 2OGDs in rice (Kawai et al. 2014) was performed using the CLUSTALX2 algorithm. Motif discovery on protein was performed using the software MEME Suite 5.2.0 (Bailey and Elkan 1994) (http://meme-suite.org/tools/meme).

\section{Protein Homology Model Building}

A homology model for HIS1 was generated using Swissmodel (https://swissmodel.expasy.org), and images were generated using PyMOL (Yuan et al. 2016).

\section{Supplementary Information}

The online version contains supplementary material available at https://doi. org/10.1186/s12284-020-00448-7.

Additional file 1: Table S1. The genetic variations of HIS1 in indica rice accessions.

Additional file 2: Table S2. PCR primers used in this study.

Additional file 3: Fig. S1. PCR-based genotyping for the 28-bp deletion in indica accessions (partially). M: DL2000 DNA marker; lane 1-24: IR64,
Nipponbare, Bing 1A, Digu, Yuetai A, 9311, Zhenxian 97A, Fengyuan A, Mianhui 725, II-32A, Tianfeng A, Fenghuazan, Yuanhui 2 hao, Yuetai B, IR36, Gui 99, Fengyuan A, Shuhui 498, Miyang 46, IR64, D62A, G46A, CDR22, Wufeng A. Fig. S2. Pfam motifs of HIS1 and amino acid variation of haplotypes. The shaded parts are predicted amino acids due to the 28-bp deletion in H5. Fig. S3. Protein sequence alignment of 2OGDs. (A) Protein sequence alignment and conserved site analysis of 91 experimentally characterized 2OGDs in plants. (B) Protein sequence alignment and conserved site analysis of 114 putative 2OGDs in rice. Fig. S4. RNA expression of HIS1 in some accessions harboring $\mathrm{H1}, \mathrm{H} 2, \mathrm{H} 3$, and $\mathrm{H} 5$. The qRT-PCR results were normalized with the actin reference gene. Error bars represent the standard error of the mean (SEM) of three replicates. Fig. S5. The T1510G mutation is completely co-segregated with the susceptibility to BBC (partially). Rice accessions Nongxiang42 and Xianhui207 carry HIS1 haplotypes $\mathrm{H} 2$ and $\mathrm{H} 3$, respectively. The upper electrophoretic band represents the PCR products of HIS1, and the lower band shows the PCR products treated with the restriction enzyme Dde1. Fig. S6. BBC is effective when the seeds are mixed. (A). BBC can effectively kill hybrid sterile lines (MingS) without harming hybrids (Mingliangyou143). The red arrow indicates the sterile line that was killed. (B). Random selection of 10 normal and severely affected seedlings. Through the detection of 28-bp deletion primers, it was found that the normal materials were all in a heterozygous state, and the sterile line materials lacking 28-bp were killed.

\section{Abbreviations}

bTH: $\beta$-triketone herbicide; BBC: Benzobicyclon; TPR: Puddled-transplanted rice; DSR: Direct seeding of rice; HPPD: 4-hydroxyphenylpyruvate dioxygenase; HIS1: HPPD INHIBITOR SENSITIVE 1; ALS: Acetolactate synthetase; ACCase: Acetyl-CoA carboxylase; P/TGMS: Photoperiod- and thermo-sensitive genic male sterile; CMS: Cytoplasm male sterile

\section{Acknowledgments}

Not applicable.

\section{Authors' Contributions}

L.B., B.Z., and L.Z. conceived and supervised this research. Q.L. and X.Z. performed most of the experiments. D.Y., Z.H., J.P., and B.M. provided most of the rice materials and genotyping. R.P., L.T., Z.L., D.L., X.Z., L.W., L.P., and $Y . X$. conducted the field experiments, performed phenotyping, and prepared the samples. Q.L., B.Z., L.B., and L.Z. wrote the manuscript. All authors read and approved the final manuscript.

Funding

This work was supported by funding from the National Key Research and Development Project of China (2016YFD0101100), Research and Innovation Fund of Hunan hybrid rice research center (ZD201901), Natural Science Foundation of Hunan Province (2018JJ3377).

\section{Availability of Data and Materials}

All data supporting the conclusions of this article are provided within the article (and its additional files).

Ethics Approval and Consent to Participate

Not applicable.

\section{Consent for Publication}

These co-authors involved in the paper all consent to publish this article in Rice.

\section{Competing Interests}

The authors declare that they have no competing interests.

\section{Author details}

${ }^{1}$ State Key Laboratory of Hybrid Rice, Hunan Hybrid Rice Research Center, Changsha, China. ${ }^{2}$ Longping Branch of Graduate School, Hunan University, Changsha, China. ${ }^{3}$ Rice Research Institute, Hunan Academy of Agricultural Sciences, Changsha, China. ${ }^{4}$ Hunan Agricultural Biotechnology Research Institute, Hunan Academy of Agricultural Sciences, Changsha, China. ${ }^{5}$ Institute of Plant Protection, Hunan Academy of Agricultural Sciences, 
Changsha, China. ${ }^{6}$ Institute of Genetics and Developmental Biology, Chinese Academy of Sciences, Beijing, China.

Received: 14 July 2020 Accepted: 17 December 2020 Published online: 07 January 2021

\section{References}

Alexandrov N, Tai S, Wang W, Mansueto L, Palis K, Fuentes RR, Ulat VJ, Chebotarov D, Zhang G, Li Z, Mauleon R, Hamilton RS, McNally KL (2015) SNP-seek database of SNPs derived from 3000 rice genomes. Ncleic Acids Res 43:D1023-D1027

Bailey TL, Elkan C (1994) "Fitting a mixture model by expectation maximization to discover motifs in biopolymers", proceedings of the second international conference on intelligent Systems for Molecular Biology. AAAI press, Menlo Park, California, pp 28-36

Beaudegnies R, Edmunds AJF, Fraser TEM, Hall RG, Hawkes TR, Mitchell G, Schaetzer J, Wendeborn S, Wibley J (2009) Herbicidal 4hydroxyphenylpyruvate dioxygenase inhibitors - a review of the triketone chemistry story from a Syngenta perspective. Bioorg Med Chem 17:41344152

Bi Y, Wang C, Gu G, Li B, Ren Y, Li X, Li S (2018) Herbicidal activity evaluation of benzobicyclon and its safety to rice. Chinese J Pesticide Sci 20:18-24

Chen L, Lei D, Tang W, Xiao Y (2011) Thoughts and practice on some problems about research and application of two-line hybrid rice. Rice Sci 18:79-85

Ding J, Lu Q, Ouyang Y, Mao H, Zhang P, Yao J, Xu C, Li X, Xiao J, Zhang Q (2012) A long noncoding RNA regulates photoperiod-sensitive male sterility, an essential component of hybrid rice. Proc Natl Acad Sci U S A 109:26542659

Farooq M, Siddique KHM, Rehman H, Aziz T, Lee D-J, Wahid A (2011) Rice direct seeding: experiences, challenges and opportunities. Soil Tillage Res 111:8798

Gould F, Brown ZS, Kuzma J (2018) Wicked evolution: can we address the sociobiological dilemma of pesticide resistance? Science 360:728-732

Hu Z, Tian Y, Xu Q (2016) Review of extension and analysis on current status of hybrid rice in China. Hybrid Rice 31:1-8

Hua N (2015) Triketone herbicide and its application. World Pesticides 37:7-13

Huang J, Li J, Zhou J, Wang L, Yang S, Hurst LD, Li W-H, Tian D (2018) Identifying a large number of high-yield genes in rice by pedigree analysis, wholegenome sequencing, and CRISPR-Cas9 gene knockout. Proc Natl Acad Sci U S A 115:E7559-E7567

Jabusch TW, Tjeerdema RS (2005) Partitioning of Penoxsulam, a new sulfonamide herbicide. J Agric Food Chem 53:7179-7183

Kawai Y, Ono E, Mizutani M (2014) Evolution and diversity of the 2-oxoglutaratedependent dioxygenase superfamily in plants. Plant J 78:328-343

Komatsubara K-I, Sekino K, Yamada Y, Koyanagi H, Nakahara S (2009) Discovery and development of a new herbicide, benzobicyclon. J Pestic Sci 34:113-114

Kraehmer H, Laber B, Rosinger C, Schulz A (2014) Herbicides as weed control agents: state of the art: I. weed control research and safener technology: the path to modern agriculture. Plant Physiol 166:1119-1131

Kumar V, Ladha JK (2011) Chapter six - direct seeding of Rice: recent developments and future research needs. In: Sparks DL (ed) Advances in Agronomy. Academic Press, pp 297-413

Luo X, Wang Z, Zeng S, Zang Y, Yang W, Zhang M (2019) Recent advances in mechanized direct seeding technology for rice. J South China Agric Univ 40: $1-13$

Mackill DJ, Khush GS (2018) IR64: a high-quality and high-yielding mega variety. Rice 11:18

Maeda H, Murata K, Sakuma N, Takei S, Yamazaki A, Karim MR, Kawata M, Hirose S, Kawagishi-Kobayashi M, Taniguchi Y, Suzuki S, Sekino K, Ohshima M, Kato H, Yoshida H, Tozawa Y (2019) A rice gene that confers broad-spectrum resistance to beta-triketone herbicides. Science 365:393-396

Mahajan G, Chauhan BS, Gill MS (2013) Dry-seeded rice culture in Punjab state of India: lessons learned from farmers. Field Crop Res 144:89-99

Moran GR (2005) 4-Hydroxyphenylpyruvate dioxygenase. Arch Biochem Biophys 433:117-128

Pan G, Zhang X, Liu K, Zhang J, Wu X, Zhu J, Tu J (2006) Map-based cloning of a novel rice cytochrome P450 gene CYP81A6 that confers resistance to two different classes of herbicides. Plant Mol Biol 61:933-943

Powles SB, Yu Q (2010) Evolution in action: plants resistant to herbicides. Annu Rev Plant Biol 61:317-347
Rao AN, Johnson DE, Sivaprasad B, Ladha JK, Mortimer AM (2007) Weed Management in direct-seeded Rice. In: Sparks DL (ed) Advances in Agronomy. Academic Press, pp 153-255

Sasaki T, International Rice Genome Sequencing, P (2005) The map-based sequence of the rice genome. Nature 436:793-800

Shoba D, Raveendran M, Manonmani S, Utharasu S, Dhivyapriya D, Subhasini G, Ramchandar S, Valarmathi R, Grover N, Krishnan SG, Singh AK, Jayaswal P, Kale P, Ramkumar MK, Mithra SVA, Mohapatra T, Singh K, Singh NK, Sarla N, Sheshshayee MS, Kar MK, Robin S, Sharma RP (2017) Development and genetic characterization of a novel herbicide (Imazethapyr) tolerant mutant in Rice (Oryza sativa L.). Rice 10:10

Sudianto E, Beng-Kah S, Ting-Xiang N, Saldain NE, Scott RC, Burgos NR (2013) Clearfield ${ }^{\oplus}$ rice: its development, success, and key challenges on a global perspective. Crop Prot 49:40-51

Tang W, Zhang G, Deng H (2020) Technology exploration and practice of hybrid Rice mechanized seed production. Chin J Rice Sci 34:95-103

Wan J, Xie H, Cheng S, Yang Q (2010) Chapter one -review and Prospect of Rice breeding. In: Chinese rice genetic breeding and variety pedigree. China agricultural press, Beijing, China, pp 1-23

Wang W, Mauleon R, Hu Z, Chebotarov D, Tai S, Wu Z, Li M, Zheng T, Fuentes RR, Zhang F, Mansueto L, Copetti D, Sanciangco M, Palis KC, XU J, Sun C, Fu B, Zhang H, Gao Y, Zhao X, Shen F, Cui X, Yu H, Li Z, Chen M, Detras J, Zhou Y, Zhang X, Zhao Y, Kudrna D, Wang C, Li R, Jia B, Lu J, He X, Dong Z, XU J, Li Y, Wang M, Shi J, Li J, Zhang D, Lee S, Hu W, Poliakov A, Dubchak I, Ulat VJ, Borja FN, Mendoza JR, Ali J, Li J, Gao Q, Niu Y, Yue Z, Naredo MEB, Talag J, Wang X, Li J, Fang X, Yin Y, Glaszmann JC, Zhang J, Li J, Hamilton RS, Wing RA, Ruan J, Zhang G, Wei C, Alexandrov N, McNally KL, Li Z, Leung H (2018) Genomic variation in 3,010 diverse accessions of Asian cultivated rice. Nature 557:43-49

Xia Y, Tang N, Hu Y, Li D, Li S, Bu X, Yu M, Qi S, Yang Y, Zhu H, Cao C, Li P, Yuan L, Cao M (2019) A method for mechanized hybrid rice seed production using female sterile rice. Rice 12:39

Xie F, Peng S (2016) History and prospects of hybrid rice development outside of China. Chin Sci Bull 61:3858-3868

Yuan S, Chan HCS, Filipek S, Vogel H (2016) PyMOL and Inkscape bridge the data and the data visualization. Structure (London, England: 1993) 24:2041-2042

Zhang J, Chen L-L, Xing F, Kudrna DA, Yao W, Copetti D, Mu T, Li W, Song J-M, Xie W, Lee S, Talag J, Shao L, An Y, Zhang C-L, Ouyang Y, Sun S, Jiao W-B, LV F, Du B, Luo M, Maldonado CE, Goicoechea JL, Xiong L, Wu C, Xing Y, Zhou D-X, Yu S, Zhao Y, Wang G, Yu Y, Luo Y, Zhou Z-W, Hurtado BEP, Danowitz A, Wing RA, Zhang Q (2016) Extensive sequence divergence between the reference genomes of two elite indica rice varieties Zhenshan 97 and Minghui 63. Proc Natl Acad Sci U S A 113:E5163-E5171

Zhang J, Xu Y, Wu X, Zhu L (2002) A bentazon and sulfonylurea sensitive mutant: breeding, genetics and potential application in seed production of hybrid rice. Theor Appl Genet 105:16-22

Zhang L, Chen H, Li Y, Li Y, Wang S, Su J, Liu X, Chen D, Chen X (2014) Evaluation of the agronomic performance of atrazine-tolerant transgenic japonica rice parental lines for utilization in hybrid seed production. PLoS One 9:e108569

Zhou H, Liu Q, Li J, Jiang D, Zhou L, Wu P, Lu S, Li F, Zhu L, Liu Z, Chen L, Liu YG, Zhuang C (2012) Photoperiod- and thermo-sensitive genic male sterility in rice are caused by a point mutation in a novel noncoding RNA that produces a small RNA. Cell Res 22:649-660

Zhou H, Zhou M, Yang Y, Li J, Zhu L, Jiang D, Dong J, Liu Q, Gu L, Zhou L, Feng M, Qin P, Hu X, Song C, Shi J, Song X, Ni E, Wu X, Deng Q, Liu Z, Chen M, Liu Y-G, Cao X, Zhuang C (2014) RNase ZS1 processes UbL40 mRNAs and controls thermosensitive genic male sterility in rice. Nat Commun 5:4884

\section{Publisher's Note}

Springer Nature remains neutral with regard to jurisdictional claims in published maps and institutional affiliations. 